\title{
HILBERT TRANSFORMS AND UNITARY EQUIVALENCE
}

\author{
by C. R. PUTNAM $\dagger$
}

(Received 4 August, 1966)

1. Introduction. If $E$ is a subset of the real line of positive measure, then the associated Hilbert transform $H=H_{E}$,

$$
(H x)(t)=(i \pi)^{-1} \int_{E}(s-t)^{-1} x(s) d s,
$$

where the integral is a Cauchy principal value, is a bounded self-adjoint operator on $L^{2}(E)$ (cf. Muskhelishvili [4]). In case $E=(-\infty, \infty)$ the transformation is also unitary with a spectrum consisting of 1 and -1 , each of infinite multiplicity (Titchmarsh [10]). If $E$ is a finite interval the spectral representation of $H$ has been given by Koppelman and Pincus [3]; see also Putnam [6]. In particular the spectrum of $H$ is in this case the closed interval $[-1,1]$. Moreover, according to Widom [11], the spectrum of $H$ is $[-1,1]$ whenever $E \neq(-\infty, \infty)$, that is, whenever

$$
(-\infty, \infty)-E \text { has positive measure. }
$$

A self-adjoint operator $A$ on a Hilbert space with the spectral resolution $A=\int \lambda d E_{\lambda}$ is said to be absolutely continuous if $\left\|E_{\lambda} x\right\|^{2}$ is an absolutely continuous function of $\lambda$ for all elements $x$ in the space. Similarly one can define an absolutely continuous unitary operator.

It was shown in Putnam [6] that $H$ of (1) is always absolutely continuous if the closure of $E$ is not $(-\infty, \infty)$, that is, if there exists some interval $J$ such that

$$
E \cap J \text { is empty. }
$$

The question as to whether $H$ is absolutely continuous if the assumption (3) is weakened to (2) apparently remains open. +

Let $\sigma(\lambda)$ denote a distribution function, so that

$$
d \sigma(\lambda) \geqq 0 \text { and } \int_{-\infty}^{\infty} d \sigma(\lambda)=1
$$

and let $k(t)$ denote its Fourier-Stieltjes transform

$$
k(t)=\int_{-\infty}^{\infty} e^{i \lambda t} d \sigma(\lambda)
$$

For any set $E$ of positive measure, define the bounded self-adjoint operator $A=A_{E}$ on $L^{2}(E)$ by

$$
(A x)(t)=(i \pi)^{-1} \int_{E} k(s-t)(s-t)^{-1} x(s) d s,
$$

† This work was supported by a National Science Foundation research grant.

$\ddagger$ Added Jan. 12, 1967: The question can now be answered affirmatively in view of recent results of $M$. Rosenblum (Amer. J. Math. 88 (1966), 314-328). 
where, as before, the integral is interpreted as a Cauchy principal value. In case $\sigma(\lambda)$ is the Dirac distribution $(\sigma(\lambda)=0$ for $\lambda<0$ and $\sigma(\lambda)=1$ for $\lambda \geqq 0)$ it is seen that $A=H$.

An investigation of the operator $A$ will be made below. The principal results are contained in the following theorem.

THEOREM 1. (i) If E satisfies (3) for some interval $J$, then $A$ of (6) is absolutely continuous. (ii) If, in addition to (3), it is also assumed that

$$
\text { meas } E<\infty \text {, }
$$

then the spectrum of $A$ is the interval $[-1,1]$. (iii) If (3), (7) and

$$
\int_{-\infty}^{\infty}|\lambda| d \sigma(\lambda)<\infty
$$

are assumed, then in fact $A$ and $H$ are unitarily equivalent; thus

$$
A=U H U^{*}
$$

for some unitary operator $U$.

Whether conditions (3) and (7) alone, without the assumption (8), are sufficient to imply (9) will remain undecided.

2. Proof of (i). Let $a$ denote any interior point of the interval $J$ satisfying (3) and let $B$ denote the bounded multiplication operator $(t-a)^{-1}$ on $L^{2}(E)$. Then $A B-B A=i C$, where $C$ is the integral operator defined by

It is seen that

$$
C x=\pi^{-1} \int_{E} k(s-t)(s-a)^{-1}(t-a)^{-1} x(s) d s .
$$

$$
(C x, x)=\pi^{-1} \int_{-\infty}^{\infty}\left|\int_{E} x(s)(s-a)^{-1} e^{i \lambda s} d s\right|^{2} d \sigma(\lambda),
$$

so that $C \geqq 0$. (What is really involved here is the fact that (5) implies the non-negative definite character of the function $k(t)$ on $(-\infty, \infty)$. The essential converse is more difficult and is due to Bochner. See Hopf [1, pp. 11-12], also Riesz and Sz.-Nagy [7, pp. 385 ff.].)

The results of Putnam [6] can now be applied to prove part (i) of Theorem 1 . Thus the absolute continuity of $A$ will be established if it is shown that $k(s-t)=0$ almost everywhere on $E \times F$, where $F$ is a measurable subset of $E$, implies that meas $F=0$. But $k(s-t)$ is clearly a continuous function of $s$ and $t$, and hence, if meas $F>0$, then $k(s-t)=0$ almost everywhere on $E \times F$ implies that

$$
k(0)\left(=\int_{-\infty}^{\infty} d \sigma(\lambda)\right)=0
$$

a contradiction to (4). This proves (i).

Part (ii) will be established as a consequence of part (iii) which will be proved next. 
3. Proof of (iii). If $g(t)$ is defined (for $t \neq 0$ ) by $k(t)=k(0)+\operatorname{tg}(t)$, so that

then

$$
g(t)=t^{-1} \int_{-\infty}^{\infty}\left(e^{i \lambda t}-1\right) d \sigma(\lambda)
$$

$$
A=H+G \text {, }
$$

where $(G x)(t)=(i \pi)^{-1} \int_{E} g(s-t) x(s) d s$. In addition,

$$
(G x, x)=2 \int_{-\infty}^{\infty} \int_{0}^{\lambda}|y(\mu)|^{2} d \mu d \sigma(\lambda),
$$

where $y(\mu)=(2 \pi)^{-\frac{1}{2}} \int_{E} x(s) e^{i \mu s} d s$ is the conjugate of the Fourier transform of $\bar{x}(s)$. (The necessary interchanges of orders of integration needed to establish (11) are certainly justified if $x(s)$ has compact support. The general case can be handled by approximations involving such elements.) Even if (3), (7) and (8) are not assumed, it is clear that (11) holds and that, further, in view of the Parseval relation

(11) implies that

$$
\int_{E}|x(s)|^{2} d s=\int_{-\infty}^{\infty}|y(\mu)|^{2} d \mu
$$

$$
\|G\| \leqq 2 k(0) \quad(=2) .
$$

(It may be noted that (12) is clearly necessary for the validity of (9). whether or not (3), (7) and (8) are assumed.)

However, if (8) is assumed and if one puts

then

$$
g(0)=i \int_{-\infty}^{\infty} \lambda d \sigma(\lambda)
$$

which is continuous and satisfies

$$
g(t)=\int_{-\infty}^{\infty}\left(i \int_{0}^{\lambda} e^{i \mu t} d \mu\right) d \sigma(\lambda)
$$

$$
|g(t)| \leqq \int_{-\infty}^{\infty}|\lambda| d \sigma(\lambda)
$$

Thus relations (7) and (8) together imply that $g(s-t)$ belongs to $L^{2}(E \times E)$, and hence that $G$ is completely continuous.

$$
\text { If } k(t)=k_{1}(t)+k_{2}(t) \text {, where }
$$

and

$$
k_{1}(t)=\int_{0}^{\infty} e^{i \lambda t} d \sigma(\lambda)
$$

$$
k_{2}(t)=\int_{-\infty}^{0-} e^{i \lambda t} d \sigma(\lambda)
$$

H 
and if one defines the associated operators $G_{1}$ and $G_{2}$ corresponding to $G$, then it is seen from relations similar to (11) that $G_{1} \geqq 0$ and $G_{2} \leqq 0$. Further (cf., e.g., Riesz and Sz.-Nagy [7, p. 245]), it is clear, in virtue of (7) and (8), that $G_{1}$ and $G_{2}$ have the finite traces

$$
\left.\pi^{-1}(\text { meas } E) \int_{0}^{\infty} \lambda d \sigma(\lambda) \text { and } \pi^{-1} \text { (meas } E\right) \int_{-\infty}^{0} \lambda d \sigma(\lambda)
$$

respectively. Therefore $G_{1}$ and $G_{2}$, and hence also $G=G_{1}+G_{2}$, are trace class operators. (For a discussion of such operators, see, e.g., Schatten [9].) Since, as has been seen above, both $A$ and $H$ are absolutely continuous, the assertion (9) is now a consequence of the RosenblumKato theory; see Rosenblum [8], Kato [2].

4. Proof of (ii). Let

$$
k_{n}(t)=\int_{-n}^{n} e^{i \lambda t} d \sigma(\lambda)
$$

for $n=1,2, \ldots$, and let $A_{n}, G_{n}$ correspond to $k_{n}$ as $A, G$ do to $k$. Then

Since, by (12),

$$
A-A_{n}=\left(k(0)-k_{n}(0)\right) H+\left(G-G_{n}\right) \text {. }
$$

$$
\left\|G-G_{n}\right\| \leqq 2\left(k(0)-k_{n}(0)\right)
$$

and since $k_{n}(0) \rightarrow k(0)(=1)$ as $n \rightarrow \infty$, it is clear that $\left\|A-A_{n}\right\| \rightarrow 0$. It follows from (iii) of Theorem 1 that $A_{n}$ is unitarily equivalent to $k_{n}(0) H$ and hence has spectrum [ $\left.-k_{n}(0), k_{n}(0)\right]$. Since $A_{n}$ and $A$ are self-adjoint, the relation $\left\|A-A_{n}\right\| \rightarrow 0$ now implies that the spectrum of $A$ is $[-1,1]$, as was to be shown.

5. Remarks. It was shown above that (3), (7) and (8) imply (9) for some unitary operator $U$. Suppose that, in addition, the spectrum of the function $\sigma(\lambda)$ is contained either in $[0, \infty)$ or in $(-\infty, 0]$; thus

$$
\sigma(\lambda)=\text { constant either for } \lambda<0 \text { or for } \lambda>0 .
$$

Then it is seen from (11) that $G$ is semi-definite. According to Putnam [5], it follows from (10) that, in this case, any unitary operator $U$ satisfying (9) is certainly absolutely continuous provided that 0 is not in the point spectrum of $G$. But if 0 is in the point spectrum of $G$, then there exists a unit element $x$ in $L^{2}(E)$ such that $G x=0$ and hence, by (11), if one also assumes $G \neq 0$, the identity

$$
\int_{E} x(s) e^{i \mu s} d s \equiv 0
$$

holds on some closed $\mu$-interval having 0 as an end-point. In case the hypotheses (3) and (7) are strengthened to

$E$ is bounded, 
then (14) implies that $x(s)=0$ almost everywhere, a contradiction. This can be seen by differentiating (14) with respect to $\mu$ and then setting $\mu=0$; thus $\int_{E} x(s) s^{n} d s=0$ for $n=0,1,2, \ldots$, from which the assertion follows.

The above results can be summarized in the following theorem.

THEOREM 2. Let (15) and (8) hold, so that (9) holds for some unitary operator U. In addition, assume that (13) holds and that $A \neq H$ (that is, $G \neq 0$ ). Then any unitary operator $U$ satisfying (9) is absolutely continuous.

\section{REFERENCES}

1. E. Hopf, Ergodentheorie, Chelsea (New York, 1948).

2. T. Kato, Perturbation of continuous spectra by trace class operators, Proc. Japan Acad. 33 (1957), 260-264.

3. W. Koppelman and J. Pincus, Spectral representations for finite Hilbert transformations, Math. Zeit. 71 (1959), 399-407.

4. N. J. Muskhelishvili, Singular integral equations (Groningen, 1953).

5. C. R. Putnam, On differences of unitarily equivalent self-adjoint operators, Proc. Glasgow Math. Assoc. 4 (1960), 103-107. 857-872.

6. C. R. Putnam, The spectra of generalized Hilbert transforms, J. Math. Mech. 14 (1965),

7. F. Riesz and B. Sz.-Nagy, Functional analysis, Ungar (New York, 1955).

8. M. Rosenblum, Perturbation of the continuous spectrum and unitary equivalence, Pacific J. Math. 7 (1957), 997-1010.

9. R. Schatten, $A$ theory of cross spaces, Ann. Math. Studies (Princeton, 1950).

10. E. C. Titchmarsh, Introduction to the theory of Fourier integrals (Oxford, 1948).

11. H. Widom, Singular integral equations in $L_{p}$, Trans. Amer. Math. Soc. 97 (1960), 131-160.

PURDUE UNIVERSITY

LAFAYETTE, INDIANA 